\title{
Sobrecarga em cuidadores de usuários de um centro de atenção psicossocial infanto-juvenil no sul do Brasil
}

\author{
Work overload faced by caregivers assisting frequenters \\ of a psychosocial care center for children and adolescents \\ in the south of Brazil
}

\author{
Clarisse de Azambuja Farias ${ }^{1}$ \\ Pedrita Oliveira Conde Lima ${ }^{1}$ \\ Lidiane Aguiar Ferreira ${ }^{1}$ \\ Ana Laura Sica Cruzeiro ${ }^{2}$ \\ Luciana de Avila Quevedo ${ }^{1}$
}

${ }^{1}$ Programa de PósGraduação em Saúde e Comportamento, Universidade Católica de Pelotas. R. Gonçalves Chaves $373 /$ sala $411 \mathrm{C}$, centro. 96015-560 Pelotas RS Brasil.

lu.quevedo@bol.com.br

${ }^{2}$ Faculdade de Psicologia, Universidade Federal de Pelotas.

\begin{abstract}
The scope of this article is to compare the work overload averages among caregivers assisting frequenters of a Psychosocial Care Center for children and adolescents (CAPSi) in Pelotas-Brazil in relation to the mental health of the children and adolescents and the caregivers themselves. It involved a cross-sectional study conducted with the primary caregiver of CAPSi frequenters. The work overload was evaluated using the Zarit Burden Interview scale and the mental health problems of frequenters were assessed using the Strengths and Difficulties Questionnaire (SDQ). To verify the presence of common potential mental disorders of caregivers, a Self-Report Questionnaire (SRQ-20) was used. T-test and ANOVA was used for data analysis. The average work overload was significantly higher in caregivers of frequenters with behavioral problems ( $p=0.000$ ), hyperactivity ( $p$ $=0.001$ ) and relationship problems with peers ( $p$ $=0.001$ ). IT was also significantly higher among those classified as potential cases of common mental disorders $(p=0.000)$; women caregivers ( $p=$ 0.032 ) and those with past problems with the Judiciary and/or Guardianship Council involving a frequenter $(p=0.039)$. The importance of work geared to caregivers to enhance the quality of care and quality of life of the caregivers themselves should be highlighted.
\end{abstract}

Key words Overload, Caregiver, Mental health, Childhood, Adolescent
Resumo O objetivo deste artigo é comparar as médias de sobrecarga de cuidadores de usuários do Centro de Atenção Psicossocial infanto-juvenil (CAPSi) da cidade de Pelotas (RS) em relação à saúde mental de crianças e adolescentes e a do próprio cuidador. Estudo transversal realizado com o principal cuidador do usuário do CAPSi. A sobrecarga foi avaliada pela escala Zarit Burden Interview e os problemas de saúde mental dos usuários através da Strengths and Difficulties Questionnaire (SDQ). Para verificar a presença de possiveis transtornos mentais comuns (TMC) nos cuidadores, utilizou-se o questionário Self-Report Questionnaire (SRQ-20). Foi utilizado o Teste te ANOVA para análise dos dados. As médias de sobrecarga foram significativamente mais altas nos cuidadores de usuários com problemas de conduta $(p=0,000)$, hiperatividade $(p=0,001)$ e problemas de relacionamento com colegas $(p=0,001)$. Também foram significativamente maiores entre aqueles classificados como possíveis casos de TMC $(p=0,000)$; entre as mulheres $(p=0,032)$ e os que já tiveram problemas na Justiça e/ou Conselho Tutelar $(p=0,039)$ envolvendo o usuário. Salienta-se a importância de trabalhos destinados aos cuidadores visando melhorar a qualidade do cuidado e da vida dos mesmos.

Palavras-chave Sobrecarga, Cuidador, Saúde mental, Infância, Adolescência 


\section{Introdução}

Nos últimos anos, vem aumentando a necessidade de espaços destinados ao cuidado da saúde mental infanto-juvenil, pois no Brasil, estima-se que de $10 \%$ a $20 \%$ de crianças e adolescentes sofrem de transtornos mentais e que de $3 \%$ a $4 \%$ necessitam de cuidado intensivo ${ }^{1}$. Assim, hoje, pode-se contar com o Centro de Atenção Psicossocial infanto-juvenil (CAPSi), o qual tem sido considerado um importante dispositivo comunitário de atenção a saúde mental de crianças e adolescentes (03 a 18 anos) portadores de sofrimento psíquico (PSP). Pode-se encontrar nesta população transtornos globais do desenvolvimento, transtornos neuróticos graves e psicóticos, bem como outros transtornos que causem prejuízos nas relações sociais ${ }^{1,2}$.

Ao mesmo tempo em que esta modalidade de assistência busca a reinserção social e a melhora da qualidade de vida do PSP, pode também acarretar sobrecarga familiar. Visto que a responsabilidade do cuidado deixou de ser exclusiva das instituições voltadas à saúde mental, passando a ser compartilhada com a família. O cuidado diário do PSP pode causar prejuízos nos familiares tanto na qualidade de vida quanto na saúde, principalmente, pelo fato de muitos cuidadores ajustarem suas rotinas em torno da doença, deixando de lado suas atividades sociais, profissionais e até mesmo o seu próprio cuidado ${ }^{3-5}$. Considera-se o cuidador principal a pessoa que pertence ao convívio familiar do PSP, a qual passa a maior parte do tempo sendo responsável pelo cuidado, exercendo este papel sem qualquer tipo de remuneração econômica ${ }^{6}$.

A sobrecarga do cuidador tem sido definida em objetiva e subjetiva. A primeira refere-se a efeitos negativos concretos decorrentes da conduta e sintomas do PSP sobre a organização habitual da vida de seu responsável. Caracteriza-se por um conjunto de efeitos adversos no que diz respeito à saúde, à situação financeira e ao convívio social, bem como perturbações nas relações entre os membros da família. Já os aspectos subjetivos incluem a percepção que o próprio cuidador faz sobre o cuidado e de como se sente emocionalmente diante desta responsabilidade ${ }^{7}$. Portanto a sobrecarga é um fator que influencia diretamente no manejo do cuidado e na qualidade de vida de quem é responsável pelo PSP8.

Estudos mostram que entre os fatores associados a maiores níveis de sobrecarga estão algumas características do próprio cuidador de PSP como: sexo, idade, escolaridade ${ }^{9}$ o grau de paren- tesco $0^{9,10}$ e a presença de transtornos mentais ${ }^{9-13}$. Contudo, outros ressaltam que a sobrecarga do cuidado, bem como o da presença de problemas de comportamento do PSP, torna os cuidadores mais suscetíveis a apresentarem sintomas depressivos e ansiosos ${ }^{14,15}$.

A literatura já evidencia a sobrecarga em cuidadores de pacientes esquizofrênicos ${ }^{5,7,16}$, com depressão $0^{11}$ e portadores de demência ${ }^{10,17}$. Estudo realizado com cuidadores de PSP que frequentam os Centros de Atenção Psicossocial (CAPS) verificou que cuidadores de usuários com esquizofrenia, retardo mental, transtorno não especificado, abuso de substâncias e transtornos de personalidade apresentaram maiores níveis de sobrecarga do que os cuidadores de usuários com transtornos neuróticos e de humor?.

No que diz respeito aos cuidadores da população infantil PSP, pesquisa direcionada a estes evidenciou que o auxílio cotidiano e preocupações com a criança estiveram relacionados à sobrecarga objetiva e subjetiva, respectivamente ${ }^{18}$. Tais evidências igualmente foram encontradas em um estudo com cuidadores de jovens adultos $\mathrm{PSP}^{16}$. Foi visto também que preocupações quanto ao futuro da criança PSP, bem como o cansaço que causam perante as suas demandas e o ressentimento na família tendem a estar relacionados ao estresse parental, no que se refere à sobrecarga emocional em cuidadores de crianças com transtorno de déficit de atenção e hiperatividade (TDAH $)^{19}$.

Diante da nova proposta de cuidados voltada para a reabilitação e reintegração social dos PSP houve um aumento no interesse de avaliar a saúde e a qualidade de vida dos cuidadores. Contudo são escassos os estudos que apontam os fatores associados à sobrecarga de cuidadores de crianças e adolescentes PSP, bem como a relação desta com os problemas de saúde mental da população infanto-juvenil. Partindo do princípio de que os familiares e/ou responsáveis são figuras fundamentais no processo de tratamento de PSP, principalmente quando o cuidado é direcionado a crianças e adolescentes, o presente estudo teve por objetivo comparar as médias de sobrecarga de cuidadores de usuários do CAPSi da cidade de Pelotas (RS) em relação à saúde mental de crianças e adolescentes e a do próprio cuidador.

\section{Métodos}

Trata-se de um estudo transversal, com cuidadores de crianças e adolescentes usuários do CAPSi. 
A seleção amostral foi realizada por conveniência, tendo como critério de inclusão entrevistar o principal cuidador das crianças e dos adolescentes, ou seja, a pessoa que fica mais tempo e que tem maior responsabilidade no cuidado dos usuários do CAPSi. O estudo teve como critério de exclusão aquelas crianças e adolescentes que estivessem vivendo em abrigos, por não terem um principal responsável, bem como os cuidadores que não conseguissem entender os instrumentos.

Os dados das crianças e dos adolescentes que estavam frequentando o CAPSi foram levantados no mês de março de 2013. Após a identificação, foi realizado contato telefônico para informar aos cuidadores sobre o objetivo do estudo e agendar as entrevistas. As entrevistadoras foram graduandas do curso de Psicologia da Universidade Católica de Pelotas (UCPel), as quais receberam treino prévio. Os encontros foram realizados em salas disponibilizadas pela coordenação do CAPSi e pela UCPel. As entrevistas foram realizadas no período de abril a maio de 2013 com todos os cuidadores ativos no CAPSi que aceitaram participar do estudo.

Para avaliar a sobrecarga dos cuidadores, utilizou-se a escala Burden Interview (BI), validada para o Brasil, a partir da versão original, por Scazufca ${ }^{11}$. Este instrumento é composto por 22 itens que avaliam a sobrecarga em relação à incapacidade funcional e comportamental da pessoa que está sendo cuidada. Possibilita que os cuidadores reflitam sobre como se sentem em relação ao cuidado no que diz respeito às áreas da saúde, vida pessoal, apoio e desempenho de atividades sociais, bem-estar emocional, relacionamento interpessoal e situação financeira.

$\mathrm{O}$ instrumento avalia a sobrecarga nas dimensões objetivas e subjetivas, contudo não fornece escores independentes. Os itens são pontuados em uma escala de 0 a 4 , sendo $0=$ nunca, 1 $=$ raramente, $2=$ algumas vezes, $3=$ frequentemente e 4 = sempre. O último item da escala refere-se quanto o cuidador se sente sobrecarregado de uma maneira geral, tendo como opções de resposta $0=$ nem um pouco, $1=$ um pouco, $2=$ moderadamente, $3=$ muito e $4=$ extremamente. As pontuações obtidas podem diversificar entre 0 a 88 pontos. A sobrecarga mais alta corresponde à maior pontuação total.

Em relação aos problemas de saúde mental das crianças e dos adolescentes que frequentavam o CAPSi no período da pesquisa, inicialmente foram consultados os diagnósticos, baseados na Classificação Internacional de Doenças (CID10), nos prontuários. Como havia certa dificul- dade com os registros, principalmente devido ao fato do diagnóstico nessa fase do desenvolvimento ser mais difícil de ser firmado, optou-se por aplicar nos cuidadores o questionário Strengths and Difficulties Questionnaire (SDQ). O instrumento foi desenvolvido por Goodman ${ }^{20}$ validado para o Brasil por Fleitlich et al. ${ }^{21}$.

O SDQ é um questionário que permite fazer o rastreamento de problemas de saúde mental de crianças e adolescentes. Avalia sintomas emocionais, problemas de conduta, hiperatividade, problemas de relacionamento com colegas e comportamento pró-social. O instrumento é composto por 25 itens ordenados em 5 escalas, contando com 5 itens cada. Há três opções de respostas que variam entre mais ou menos verdadeiro, a qual geralmente é calculada como 1 , verdadeiro e falso. Ambas as últimas variam o seu valor de acordo com o item. A pontuação para cada uma das 5 escalas pode variar entre 0 e 10. Quanto maior a pontuação nas escalas, maior o número de queixas, exceto na de comportamento pró-social. Todas as escalas são somadas, permitindo a classificação da criança em três categorias: Desenvolvimento Normal (DN), Limítrofe (DL) ou Anormal (DA). Devido à escala de comportamento pró-social não computar no escore de dificuldades totais, optou-se por não utilizá -la. Para análise dos dados optou-se em categorizar ausência de problemas (normal/limítrofe) e presença de problemas (anormal).

A saúde mental dos cuidadores foi avaliada através do Self-Report Questionnaire (SRQ-20), o qual foi validado para o Brasil, por Mari e Wiliams ${ }^{22}$. O instrumento possibilita fazer o rastreamento de transtornos mentais comuns como: depressão, ansiedade, distúrbios somatoformes e neurastenia. É composto por 20 itens, cada um destes apresentam duas opções de respostas referentes a concordar ou não (escores 0 e 1) com a presença de certas dores e problemas nos últimos 30 dias. As pontuações podem variar de 0 a 20 , sendo que escores $\geq 8$, indicam possíveis casos de transtornos mentais comuns.

Além dos instrumentos citados, os cuidadores responderam a um questionário com variáveis sociodemográficas (sexo, idade, estado civil, estar trabalhando, número de filhos), socioeconômica, comportamentais (uso de álcool e tabaco), de saúde e relacionadas ao cuidado com o usuário. Para avaliar a classificação econômica foram utilizados os critérios da Associação Brasileira de Empresas de Pesquisa (ABEP) ${ }^{23}$, os quais são baseados no acúmulo de bens materiais e na escolaridade do chefe da família, classificando em 
cinco níveis (A, B, C, D e E), sendo A o nível mais alto e $\mathrm{E} o$ mais baixo.

Após a coleta de dados, estes foram duplamente digitados no programa Epi Info 6.04 para comparação e eliminação de inconsistências. O pacote estatístico SPSS 13.0 foi utilizado para a análise dos dados. A análise univariada foi realizada para descrever as características da amostra. A sobrecarga (BI) foi analisada através da comparação das médias utilizando-se o Teste t e ANOVA.

Todos os cuidadores que participaram do estudo assinaram um termo de consentimento livre e esclarecido, afirmando terem conhecimentos sobre a finalidade da pesquisa, sendo também assegurados quanto à confidencialidade de seus dados. Os cuidadores que foram classificados como possíveis casos de transtornos mentais comuns foram encaminhados para o Ambulatório de Pesquisas em Saúde Mental do Programa de Pós-graduação em Saúde e Comportamento da UCPel. Já os que estavam muito sobrecarregados foram orientados a procurarem a Unidade Básica de Saúde próxima ao seu domićlio. O projeto foi aprovado pelo Comitê de Ética da UCPel.

\section{Resultados}

Foram levantados os dados de 131 prontuários de crianças e adolescentes que estavam no registro de usuários do CAPSi. Dos 131, foram excluídos 7 (5,3\%) por viverem em abrigos e 23 $(17,5 \%)$ por já estarem desligados do serviço no período do levantamento. Resultando assim em 101 cuidadores a serem entrevistados. Destes cuidadores de usuários ativos no serviço do CAPSi, $7(6,9 \%)$ se recusaram a participar do estudo e 4 $(3,9 \%)$ foram perdas (falta de contato). Portanto a amostra total foi constituída por 90 cuidadores.

Em relação às características dos cuidadores, $95,6 \%$ eram mulheres; com idades entre 25 a 70 anos (média $43,1 \mathrm{dp} \pm 10,3$ ); 38,9\% eram casados ou viviam com o companheiro; $60 \%$ pertenciam à classe econômica $\mathrm{C}$ e $66,7 \%$ não trabalhavam. Quanto ao número de filhos $36 \%$ tinham de 4 a 8 filhos. A pontuação média na escala de sobrecarga dos cuidadores foi de 37,4 (dp $\pm 16,7)$. Esta foi significativamente maior entre as mulheres $(\mathrm{p}=$ 0,032) (Tabela 1).

A Tabela 2 refere-se às características dos cuidadores em relação aos usuários do CAPSi. Do total de cuidadores, $80 \%$ eram mães; $94,4 \%$ eram familiares. Em relação ao cuidado, $61,1 \%$ tinham mais dependentes além do usuário; $48,9 \%$ cui- davam de quatro ou mais pessoas e $53,3 \%$ contavam com a ajuda de mais alguma pessoa no cuidado da criança ou adolescente PSP. As médias de sobrecarga não apresentaram diferenças significativas entre tais variáveis.

Quanto às características comportamentais e de saúde dos cuidadores, dos 90 participantes, $73,3 \%$ fumavam mais de 20 cigarros por dia e $12,2 \%$ ingeriam bebida alcoólica. No que diz respeito à saúde em geral $62,2 \%$ dos entrevistados mencionaram ter problemas de saúde. Em relação à saúde mental, $68,9 \%$ relataram problemas mentais; $34,4 \%$ já fizeram tratamento com psicólogo e/ou psiquiatra e 38,9\% usavam psicofármacos. Verificou-se que $66,7 \%$ participantes apresentaram possíveis casos de transtornos mentais comuns e que a média de sobrecarga foi significativamente maior entre estes $(p=0,000)$. (Tabela 3)

Em relação às características das crianças e dos adolescentes, observou-se que as idades variaram entre 6 e 18 anos, sendo 12,1 a média de idade $(\mathrm{dp} \pm 3,1)$. Quanto à escolaridade $96,7 \%$ estavam estudando; sendo que $55,6 \%$ encontravam-se entre $1^{\text {a }}$ e $4^{\mathrm{a}}$ série. Do total de usuários, $34,4 \%$ já tiveram algum problema na Justiça e/ ou Conselho Tutelar; $48,3 \%$ tinham sintomas emocionais; $77,8 \%$ problemas de conduta; $84,3 \%$ hiperatividade e $56,2 \%$ problemas de relacionamento com colegas. As médias de sobrecarga foram significativamente maiores nos cuidadores que tiveram problemas na Justiça e/ou Conselho Tutelar envolvendo a criança ou adolescente ( $\mathrm{p}=$ $0,039)$ e entre aqueles que faziam o cuidado de usuários com problemas de conduta $(\mathrm{p}=0,000)$, hiperatividade $(\mathrm{p}=0,001)$ e problemas de relacionamento com colegas ( $\mathrm{p}=0,001)$. (Tabela 4 )

\section{Discussão}

De acordo com os sintomas e os comportamentos verificados nos usuários do CAPSi da cidade de Pelotas, os transtornos do comportamento e os emocionais foram identificados como os mais predominantes nos registros de sete unidades do CAPSi, distribuídas em alguns estados do Brasi $^{24}$. No presente estudo observou-se que os cuidadores de crianças e adolescentes com sintomas de hiperatividade, problemas de conduta e de relacionamento com colegas apresentaram maiores níveis de sobrecarga do que os cuidadores de usuários que portavam sintomas emocionais. Visto que os sintomas de hiperatividade e problemas de conduta se expressam de forma ex- 
Tabela 1. Características sócio demográficas e médias de sobrecarga de cuidadores de usuários do CAPSi. Pelotas-RS, 2013.

\begin{tabular}{|c|c|c|c|}
\hline Variáveis & $\mathrm{N}(\%)$ & Média BI (desvio padrão) & p-valor \\
\hline Sexo & & & 0,032 \\
\hline Feminino & $86(95,6)$ & $38,2( \pm 16,6)$ & \\
\hline Masculino & $4(4,4)$ & $20,0( \pm 5,2)$ & \\
\hline Idade & & & 0,141 \\
\hline De 25 a 34 & $22(24,4)$ & $36,4( \pm 13,6)$ & \\
\hline De 35 a 43 & $25(27,8)$ & $36,6( \pm 19,3)$ & \\
\hline De 44 a 50 & $22(24,4)$ & $43,9( \pm 17,4)$ & \\
\hline De 51 a 70 & $21(23,3)$ & $32,2( \pm 13,9)$ & \\
\hline Estado Civil & & & 0,382 \\
\hline Solteiro & $32(35,6)$ & $37,7( \pm 13,4)$ & \\
\hline Casado/ vive junto & $35(38,9)$ & $34,4( \pm 18,4)$ & \\
\hline Divorciado & $16(17,8)$ & $43,2( \pm 16,8)$ & \\
\hline Viúvo & $7(7,8)$ & $36,4( \pm 20,5)$ & \\
\hline Classe Econômica & & & 0,068 \\
\hline $\mathrm{B}$ & $5(5,6)$ & $21,2( \pm 0,8)$ & \\
\hline $\mathrm{C}$ & $54(60,0)$ & $37,5( \pm 17,8)$ & \\
\hline $\mathrm{D}+\mathrm{E}$ & $31(34,4)$ & $39,7( \pm 14,5)$ & \\
\hline Trabalha atualmente & & & 0,607 \\
\hline Não & $60(66,7)$ & $38,0( \pm 16,0)$ & \\
\hline Sim & $30(33,3)$ & $36,0( \pm 18,1)$ & \\
\hline Número de filhos & & & 0,413 \\
\hline De um a dois & $31(34,8)$ & $39,9( \pm 15,6)$ & \\
\hline Três & $26(29,2)$ & $36,8( \pm 16,8)$ & \\
\hline De quatro a oito & $32(36,0)$ & $34,3( \pm 17,1)$ & \\
\hline Total & $90(100)$ & $37,4( \pm 16,7)$ & \\
\hline
\end{tabular}

Tabela 2. Características relacionadas ao cuidado e médias de sobrecarga de cuidadores de usuários do CAPSi. Pelotas-RS, 2013.

\begin{tabular}{|c|c|c|c|}
\hline Variáveis & N (\%) & Média BI (desvio padrão) & p-valor \\
\hline Cuidador Principal & & & 0,174 \\
\hline Mãe & $72(80,0)$ & $38,6( \pm 16,8)$ & \\
\hline Outros & $18(20,0)$ & $32,6( \pm 15,5)$ & \\
\hline Tipo de relação com a criança & & & 0,569 \\
\hline Familiar & $85(94,4)$ & $37,6( \pm 16,8)$ & \\
\hline Não familiar & $5(5,6)$ & $33,2( \pm 15,4)$ & \\
\hline Tem mais pessoas que dependem do cuidador & & & 0,385 \\
\hline Não & $35(38,9)$ & $35,4( \pm 17,9)$ & \\
\hline Sim & $55(61,1)$ & $38,6( \pm 15,9)$ & \\
\hline Número de dependentes & & & 0,111 \\
\hline Um & $19(34,5)$ & $41,3( \pm 15,2)$ & \\
\hline Dois & $14(23,5)$ & $44,0( \pm 11,4)$ & \\
\hline Três & $13(23,6)$ & $38,4( \pm 18,0)$ & \\
\hline Quatro ou mais & $44(48,9)$ & $33,3( \pm 17,6)$ & \\
\hline Tem alguém que ajuda o cuidador a fazer o cuidado & & & 0,097 \\
\hline Não & $42(46,7)$ & $40,5( \pm 17,0)$ & \\
\hline Sim & $48(53,3)$ & $34,6( \pm 16,1)$ & \\
\hline Total & $90(100)$ & $37,4( \pm 16,7)$ & \\
\hline
\end{tabular}


Tabela 3. Características comportamentais e de saúde e médias de sobrecarga de cuidadores usuários do CAPSi. Pelotas-RS, 2013.

\begin{tabular}{|c|c|c|c|}
\hline Variáveis & $\mathbf{N}(\%)$ & Média BI (desvio padrão) & p-valor \\
\hline Quantidade de cigarros que fuma por dia & & & 0,400 \\
\hline Até cinco & $7(7,8)$ & $29,7( \pm 6,6)$ & \\
\hline De 6 a 12 & $8(8,9)$ & $31,8( \pm 11,6)$ & \\
\hline De 13 a 20 & $9(10)$ & $40,7( \pm 11,8)$ & \\
\hline Mais de 20 & $66(73,3)$ & $38,4( \pm 18,2)$ & \\
\hline Bebe & & & 0,422 \\
\hline Não & $79(87,8)$ & $37,9( \pm 16,8)$ & \\
\hline Sim & $11(12,2)$ & $33,5( \pm 15,8)$ & \\
\hline Problemas de saúde em geral & & & 0,242 \\
\hline Não & $34(37,8)$ & $34,7( \pm 17,7)$ & \\
\hline Sim & $56(62,2)$ & $39,0( \pm 16,0)$ & \\
\hline Problemas de nervos & & & 0,088 \\
\hline Não & $28(31,1)$ & $32,9( \pm 15,8)$ & \\
\hline Sim & $62(68,9)$ & $39,4( \pm 16,8)$ & \\
\hline Já fez tratamento com psicólogo e/ou psiquiatra & & & 0,895 \\
\hline Não & $59(65,6)$ & $37,1( \pm 2,3)$ & \\
\hline Sim & $31(34,4)$ & $37,6( \pm 2,4)$ & \\
\hline Toma medicação para problemas de nervos & & & 0,164 \\
\hline Não & $55(61,1)$ & $35,4( \pm 15,3)$ & \\
\hline Sim & $35(38,9)$ & $40,4( \pm 18,5)$ & \\
\hline Transtornos Mentais Comuns & & & 0,000 \\
\hline Não & $30(33,3)$ & $23,8( \pm 9,3)$ & \\
\hline Sim & $60(66,7)$ & $44,1( \pm 15,4)$ & \\
\hline Total & $90(100)$ & $37,4( \pm 16,7)$ & \\
\hline
\end{tabular}

ternalizada, por meio de atos comportamentais, muitas vezes relacionados à agressividade, podese pensar que tal externalização acarreta maior impacto negativo sobre o ambiente ${ }^{25}$ e maior nível de sobrecarga do cuidador.

Corroborando com os resultados encontrados, um estudo destinado a cuidadores da população infantil evidenciou que cuidadores de crianças com TDAH combinado e com TDAH e comorbidade com o Transtorno Opositor Desafiador (TOD) apresentaram mais estresse parental quando comparados com os cuidadores de crianças sem tais problemas. Sendo a sobrecarga emocional a dimensão de estresse que apresentou média mais alta entre os cuidadores de crianças com problemas de comportamento. O estudo verificou também que quanto maior a severidade dos sintomas de hiperatividade maior a sobrecarga emocional ${ }^{19}$.

Em relação aos problemas com colegas, muitas crianças e adolescentes PSP sofrem por não se enturmarem, podendo gerar certa resistência quanto ao ambiente escolar e dificuldades nos demais relacionamentos interpessoais. Isto pode acarretar a necessidade de auxílio cotidiano, provocando maior grau de dependência, bem como preocupações e maior sobrecarga do cuidador ${ }^{9,18}$. No que diz respeito à presença de sintomas emocionais, a prevalência destes foi menor comparada aos demais problemas avaliados. Pode-se pensar que pelo fato de tais sintomas serem mais internalizados, os quais são mais facilmente percebidos pelos próprios sujeitos, sem muita manifestação comportamental ${ }^{25}$, seja possível que muitos cuidadores não tenham os identificado com tanta clareza, bem como não tenham sentido um grande impacto destes em suas vidas.

Contudo, outra pesquisa destinada a verificar possíveis problemas na saúde mental em escolares de Ribeirão Preto (SP) evidenciou que de acordo com os pais os sintomas emocionais predominaram mais entre os escolares do que os problemas de conduta, hiperatividade e problemas de relacionamento interpessoal ${ }^{26}$. A diferença entre as percepções dos pais de escolares e dos cuidadores do presente estudo, pode ser pensada devido a maior intensidade das manifestações de comportamentos problemáticos dos usuários do 
Tabela 4. Características dos usuários do CAPSi e médias de sobrecarga dos cuidadores. Pelotas-RS, 2013.

\begin{tabular}{|c|c|c|c|}
\hline Variáveis & $\mathbf{N}(\%)$ & Média BI (desvio padrão) & p-valor \\
\hline Idade & & & 0,879 \\
\hline 6 a 10 anos & $28(31,1)$ & $37,0( \pm 16,2)$ & \\
\hline 11 a 12 anos & $19(21,1)$ & $37,1( \pm 14,1)$ & \\
\hline 13 a 15 anos & $31(34,4)$ & $39,0( \pm 19,0)$ & \\
\hline 16 anos ou mais & $12(13,3)$ & $34,4( \pm 16,8)$ & \\
\hline A criança/adolescente estuda & & & 0,447 \\
\hline Não & $3(3,3)$ & $54,7( \pm 33,0)$ & \\
\hline Sim & $87(96,7)$ & $36,8( \pm 15,9)$ & \\
\hline Série & & & 0,699 \\
\hline $1^{\mathrm{a}} \mathrm{a} 4^{\mathrm{a}}$ & $50(55,6)$ & $36,8( \pm 15,5)$ & \\
\hline $5^{\mathrm{a}}$ a $8^{\mathrm{a}}$ & $37(41,1)$ & $38,7( \pm 18,2)$ & \\
\hline $2^{\circ}$ grau & $3(3,3)$ & $31,0( \pm 19,1)$ & \\
\hline Problemas na Justiça ou Conselho Tutelar & & & 0,039 \\
\hline Não & $59(65,6)$ & $34,7( \pm 16,9)$ & \\
\hline Sim & $31(34,4)$ & $42,4( \pm 15,4)$ & \\
\hline Sintomas emocionais & & & 0,207 \\
\hline Não & $45(51,7)$ & $35,6( \pm 18,6)$ & \\
\hline Sim & $42(48,3)$ & $40,1( \pm 14,4)$ & \\
\hline Problemas de conduta & & & 0,000 \\
\hline Não & $20(22,2)$ & $24,2( \pm 9,6)$ & \\
\hline Sim & $70(77,8)$ & $41,1( \pm 16,4)$ & \\
\hline Hiperatividade & & & 0,001 \\
\hline Não & $14(15,7)$ & $25,1( \pm 11,4)$ & \\
\hline Sim & $75(84,3)$ & $39,9( \pm 16,5)$ & \\
\hline Problemas de relacionamento com colegas & & & 0,001 \\
\hline Não & $39(42,8)$ & $31,0( \pm 14,2)$ & \\
\hline Sim & $50(56,2)$ & $42,6( \pm 16,9)$ & \\
\hline Total & $90(100)$ & $37,4( \pm 16,7)$ & \\
\hline
\end{tabular}

CAPSi. Exigindo, assim, mais atenção dos cuidadores diante das possíveis consequências dos sintomas externalizados.

No presente estudo verificou-se que $66,7 \%$ dos cuidadores foram classificados como possíveis casos de transtornos mentais comuns e que a média de sobrecarga destes foi significativamente maior ao ser comparada com a dos que não foram classificados. Estes resultados são consistentes com a literatura que aponta que a presença de morbidades psiquiátricas nos cuidadores de crianças e adolescentes PSP está estatisticamente associada à sobrecarga ${ }^{13}$. Corroborando com estudos destinados a cuidadores de adultos jovens e adultos PSP $^{9-12}$.

Observou-se também neste estudo, que as mulheres apresentaram maiores níveis de sobrecarga. Além de estas comporem a maior parte dos cuidadores, o que também foi reforçado em outros estudos ${ }^{5,9-13,16-18}$. Percebe-se que a demanda do cuidado principal ainda está direcionada às mulheres, o que contribui para o aumento da responsabilidade, tempo de dedicação, acarretando maiores níveis de sobrecarga ${ }^{9,18,27}$. Diante deste resultado, torna-se importante que haja maior participação da família para que o cuidado seja compartilhado, evitando possíveis efeitos danosos à qualidade de vida das mulheres ${ }^{28}$.

Outro resultado importante encontrado neste estudo foi que os cuidadores que tiveram problemas na Justiça e/ou Conselho Tutelar, envolvendo a criança ou adolescente, apresentaram média de sobrecarga significativamente maior do que os que não relataram tais problemas. Contudo, o presente estudo não descreveu os motivos que levaram a ocorrência destes problemas. Não foi encontrada na literatura tal associação, o que nos leva a pensar na necessidade de estudos futuros, destinados a este tema e a esta população de cuidadores.

O presente estudo apresenta algumas limitações a serem destacadas. Por se tratar de um estudo transversal não podemos fazer inferências causais. Não temos como afirmar se os transtornos mentais comuns foram desencadeados devido à sobrecarga do cuidado ou se a existência dos transtornos mentais comuns acarretou maior 
sobrecarga. Estudos longitudinais poderão contribuir positivamente para maior compreensão sobre a direção em que os fenômenos ocorrem, tal como considerado por outros autores ${ }^{14}$.

Em relação aos resultados, estes não podem ser generalizados à população geral de cuidadores de PSP, pois a amostra foi limitada aos responsáveis de usuários do CAPSi. No que diz respeito ao rastreamento de problemas de saúde mental das crianças e dos adolescentes, o presente estudo contou apenas com as informações obtidas pelo principal cuidador, podendo haver um viés na confirmação destas. Fazendo-se necessário mais de um informante, além de um método para combinar as informações.

Apesar das limitações, pode-se considerar que o presente estudo revela a importância de investigações relacionadas aos cuidadores de PSP. Destaca-se a necessidade de haver nos serviços de saúde mental coletiva a construção de dispositivos que possibilitem o apoio e que favoreçam a participação da família, desenvolvendo também trabalhos destinados a avaliarem o impacto do cuidado sobre a vida do cuidador e as suas necessidades. Além de proporcionarem orientações quanto à saúde, visto a alta prevalência de possíveis casos de transtornos mentais comuns e de tabagismo.

Quanto ao cuidado, torna-se pertinente a realização de grupos de familiares, visando proporcionar esclarecimentos sobre as formas de manejo do cuidado em relação aos transtornos mentais. Tal como a elaboração de estratégias que auxiliem os cuidadores a estimular as capacidades de socialização das crianças e adolescentes no âmbito familiar, o que irá contribuir para uma orientação diante aos comportamentos e o desenvolvimento da autonomia ${ }^{29}$. Estas e as demais formas de intervenções poderão contribuir para melhorar a qualidade do cuidado e da vida dos cuidadores. Os resultados deste estudo se tornam relevantes para os serviços e políticas de saúde mental, salientando a importância de apoiar e de estar atento à saúde dos cuidadores.

\section{Colaboradores}

CA Farias trabalhou na elaboração do estudo, revisão bibliográfica, coleta de dados da pesquisa, análise e intepretação dos dados, bem como na elaboração do artigo e na revisão da versão a ser publicada. POC Lima trabalhou na concepção do estudo, metodologia, coleta de dados e na revisão bibliográfica. L Ferreira, trabalhou na pesquisa e na revisão da versão a ser publicada. ALS Cruzeiro trabalhou na orientação da elaboração do estudo. Quevedo LA trabalhou na orientação e elaboração do estudo, revisão bibliográfica, análise e intepretação dos dados, bem como na elaboração do artigo e na revisão da versão a ser publicada. 


\section{Referências}

1. Brasil. Ministério da Saúde (MS). Caminhos para uma política de saúde mental infanto-juvenil. Secretaria de Atenção à Saúde. Departamento de Ações Programáticas Estratégicas. [Série B. Textos Básicos em Saúde na internet]. Brasília; 2005. [acessado 2012 nov 12]; [76 p.]. Disponível em: http://portal.saude.gov.br/portal/ arquivos/pdf/caminhos_infantojuv.pdf

2. Brasil. Ministério da Saúde (MS). Saúde mental no SUS: os centros de atenção psicossocial. Secretaria de Atenção à Saúde. Departamento de Ações Programáticas Estratégicas. [Série F. Comunicação e Educação em Saúde na internet]. Brasília; 2004. [acessado 2012 out 12]; [86 p.]. Disponível em: http://www.ccs.saude.gov. br/saude_mental/pdf/sm_sus.pdf

3. Miles A. A família do doente mental. In: Miles A. $O$ doente mental na sociedade contemporânea. Rio de Janeiro: Zahar; 1982. p. 111-134.

4. Melman J. Quem são esses familiares? In: Melman J. Família e doença mental: repensando a relação entre profissionais de saúde e familiares. São Paulo: Escrituras; 2006. p. 19-38.

5. Teixeira MB. Qualidade de vida de familiares cuidadores do doente esquizofrênico. Rev Bras Enferm 2005; 58(2):171-175.

6. Dwyer J, Lee G, Jankowski T. Reciprocity, Elder Satisfaction and Caregiver Stress and Burden: The Exchange of Aid in the Family Caregiving Relationship. J Marriage Fam 1994; 56(1):35-43.

7. Hoenig J, Hamilton MW. The schizophrenic patient in the community and his effect on the household. Int $J$ Soc Psychiatry 1966; 12(3):165-176.

8. Santos RL, Souza MFB, Brasil D, Dourado M. Intervenções de grupo para sobrecarga de cuidadores de pacientes com demência: uma revisão sistemática. Rev Psiquiatr Clín 2011; 38(4):161-167.

9. Tomasi E, Rodrigues JO, Feijó GP, Facchini LA, Piccini RX, Thumé E, Da Silva RA, Gonçalves H. Sobrecarga em familiares de portadores de sofrimento psíquico que frequentam Centros de Atenção Psicossocial. Saúde em Debate 2010; 34(84):159-167.

10. Garrido R, Menezes PR. Impacto em cuidadores de idosos com demência atendidos em um serviço psicogeriátrico. Rev Saude Publica 2004; 38(6):835-841.

11. Scazufca M. Brazilian version of the Burden Interview scale for the assessment of burden of care in careers of people with mental illness. Rev Bras Psiquiatr 2002; 24:12-17.

12. Quadros LCM, Gigante DP, Kantorski LP, Jardim VMR. Transtornos psiquiátricos menores em cuidadores familiares de usuários de Centros de Atenção Psicossocial do Sul do Brasil. Cad Saude Publica 2012; 28(1):95-103.

13. Dada MU, Okewole NO, Ogun OC, Bello-Mojeed MA. Factors associated with caregiver burden in a child and adolescent psychiatric facility in Lagos, Nigeria: a descriptive cross sectional study. BMC Pediatr 2011; 11:2-6.

14. Bandeira M, Barroso SM. Sobrecarga das famílias de pacientes psiquiátricos. J Bras Psiquiatr 2005; 54(1):34-46.

15. Garrido R, Almeida OP. Distúrbios de comportamento em pacientes com demência: impacto sobre a vida do cuidador. Arq Neuropsiquiatr 1999; 57(2B):427-434.
16. Barroso SM, Bandeira M, Nascimento E. Sobrecarga de familiares de pacientes psiquiátricos atendidos na rede pública. Rev Psiq Clín 2007; 34(6):270-277.

17. Taub A, Andreoli SB, Bertolucci P H. Dementia caregiver burden: reliability of the Brazilian version of the Zarit caregiver burden interview. Cad Saude Publica 2004; 20(2):372-376.

18. Marini AM, Martins MRI, Vigãno A, Marques Filho AB, Pontes HER. Sobrecarga de cuidadores na psiquiatria infantil. Rev Neurocienc 2010; 18:300-306.

19. Bellé AH, Andreazza AC, Ruschel J, Bosa CA. Estresse e Adaptação Psicossocial em Mães de Crianças com Transtorno de Déficit de Atenção/Hiperatividade. Psicol Refl Crít 2009; 22(3):317-325.

20. Goodman R. The Strengths and Difficulties Questionnaire: a research note. J Child Psychol Psychiatry 1997; 38(5):581-586.

21. Fleitlich B, Cortázar PG, Goodman R. Questionário de capacidades e dificuldades (SDQ). Infanto-Rev Neuropsiq da Infe Adol 2000; 8:44-50.

22. Mari JJ, Williams PA. Validity study of a psychiatric screening questionnaire (SRQ-20) in primary care in the city of Sao Paulo. Br J Psych 1986; 148:23-26.

23. Associação Brasileira de Empresas de Pesquisa (ABEP). Dados com base no Levantamento Sócio Econômico (IBOPE). 2010 [acessado 2012 nov 14]. Disponível em: http://www.abep.org

24. Hoffmann MCCL, Santos DN, Mota ELA. Caracterização dos usuários e dos serviços prestados por Centros de Atendimento Psicossocial Infanto-Juvenil. Cad Saude Publica 2008; 24(3):633-642.

25. Sousa MRC, Moraes C. Sintomas de internalização e externalização em crianças e adolescentes com excesso de peso. J Bras Psiquiatr 2011; 60(1):40-45.

26. Cury C R, Golfeto J H. Strengths and difficulties questionnaire (SDQ): a study of school children in Ribeirão Preto. Rev Bras Psiquiatr 2003; 25(3):139-145.

27. Pegoraro RF, Caldana RHL. Mulheres, Loucura e Cuidado: a condição da mulher na provisão e demanda por cuidados em saúde mental. Saúde Soc 2008; 17(2):82-94.

28. Gutierrez DMD, Minayo MCS. Produção de conhecimento sobre cuidados da saúde no âmbito da família. Cien Saude Colet 2010; 15(Supl. 1):1497-1508.

29. Bigras M, Machado AL. Apontamentos e reflexões sobre programas de apoio familiar que favorecem a competência social da criança. Cien Saude Colet 2014; 19(3):663-672.

Artigo apresentado em 08/10/2013

Aprovado em 26/04/2014

Versão final apresentada em 02/05/2014 
